\title{
Multiplexed ISSR genotyping by sequencing distinguishes two precious coral species (Anthozoa: Octocorallia: Coralliidae) that share a mitochondrial haplotype
}

\author{
Kenji Takata ${ }^{1,2}$, Hiroki Taninaka ${ }^{3}$, Masanori Nonaka ${ }^{4}$, Fumihito Iwase $^{5}$, Taisei Kikuchi ${ }^{6}$, Yoshihisa Suyama $^{7}$, \\ Satoshi Nagai ${ }^{8}$, Nina Yasuda ${ }^{\text {Corresp. } 2}$ \\ ${ }^{1}$ Graduate School of Agriculture, Faculty of Agriculture, University of Miyazaki, Miyazaki, Miyazaki, Japan \\ Department of Marine Biology and Environmental Sciences, Faculty of Agriculture, University of Miyazaki, Miyazaki, Miyazaki, Japan \\ 3 Interdisciplinary Graduate School of Agriculture and Engineering, University of Miyazaki, Miyazaki, Miyazaki, Japan \\ 4 Okinawa Churashima Foundation Reseach Center, Motobu, Okinawa, Japan \\ 5 Shikoku Marine Life Laboratory, Otsuki, Kochi, Japan \\ 6 Parasitology, Faculty of Medicine, University of Miyazaki, Miyazaki, Miyazaki, Japan \\ 7 Field Science Center, Graduate School of Agricultural Science, Tohoku University, Osaki, Miyagi, Japan \\ 8 Research Centre for Bioinformatics and Biosciences Environmental genomics group, Japan Fisheries Research and Education Agency, National Research \\ Institute of Fisheries Science, Yokohama, Kanagawa, Japan \\ Corresponding Author: Nina Yasuda \\ Email address: nina27@cc.miyazaki-u.ac.jp
}

Background: Precious corals known as coralliid corals (Anthozoa: Octocorallia), play an important role in increasing the biodiversity of the deep sea. Currently, these corals are highly threatened because of overfishing that has been brought on by an increased demand and elevated prices for them. The deep sea precious corals Pleurocorallium elatius and Pleurocorallium konojoi are co-distributed in Japanese waters and have distinct morphological features: (1) the terminal branches of the colony form of $P$. elatius are very fine, while those of $P$. konojoi are blunt and rounded, (2) the autozooids of $P$. elatius are arranged in approximately four rows, while those of $P$. konojoi are clustered in groups. However, previous genetic analysis using mtDNA and nuclear DNA did not indicate monophyly. Therefore, it is important to clarify their species status to allow for their conservation.

Methodology: We collected a total of 87 samples (60 of $C$. japonicum and 27 of $P$. konojoi) from around the Ryukyu Islands and Shikoku Island, which are geographically separated by approximately $1300 \mathrm{~km}$. We used a multiplexed inter-simple sequence repeat (ISSR) genotyping by sequencing (MIG-seq) and obtained 223 SNPs with which to perform STRUCTURE analysis and principle coordinate analysis (PCoA). In addition, two relatively polymorphic mtDNA regions were sequenced and compared.

Results: Pleurocorallium elatius and $P$. konojoi share a same mtDNA haplotype, which has been previously reported. However, MIG-seq analysis clearly distinguished the two species based on PCoA and STRUCTURE analysis, including $5 \%$ of species-specific fixed SNPs.

Conclusion: This study indicated that $P$. elatius and $P$. konojoi are different species and therefore both species should be conserved separately. Our findings highlight the importance of the conservation of these two species, especially $P$. elatius, whose population has been dramatically depleted over the last 100 years. The study also demonstrated the effectiveness and robustness of MIG-seq for defining closely related octocoral species that were otherwise indistinguishable using traditional genetic markers (mtDNA and $\mathrm{EF}$ ). 
1 Multiplexed ISSR genotyping by sequencing distinguishes two precious coral

2 species (Anthozoa: Octocorallia: Coralliidae) that share a mitochondrial

3 haplotype

4

5 Kenji Takata ${ }^{1,8}$, Hiroki Taninaka ${ }^{2}$, Masanori Nonaka ${ }^{3}$, Fumihito Iwase ${ }^{4}$, Taisei Kikuchi ${ }^{5}$,

6 Yoshihisa Suyama $^{6}$, Satoshi Nagai ${ }^{7}$, Nina Yasuda ${ }^{8 *}$

$8{ }^{1}$ Graduate School of Agriculture, Faculty of Agriculture, University of Miyazaki, Miyazaki 889-

9 2192, Japan

$10{ }^{2}$ Interdisciplinary Graduate School of Agriculture and Engineering, University of Miyazaki,

11 Miyazaki 889-2192, Japan

$12{ }^{3}$ Okinawa Churashima Foundation Reseach Center, Ishikawa Motobu-Cho,

13 Okinawa 905-0206, Japan

$14{ }^{4}$ Shikoku Marine Life Laboratory, Otsuki, Kochi 788-0333, Japan.

$15{ }^{5}$ Parasitology, Faculty of Medicine, University of Miyazaki, Miyazaki 889-1692, Japan

$16{ }^{6}$ Field Science Center, Graduate School of Agricultural Science, Tohoku University, Yomogida,

17 Naruko-onsen, Osaki, Miyagi 989-6711, Japan

$18{ }^{7}$ Japan Fisheries Research and Education Agency, National Research Institute of Fisheries

19 Science, Research Centre for Bioinformatics and Biosciences Environmental genomics group,

20 Fukuura, Kanazawa-ku, Yokohama, Kanagawa 236-8648, Japan

$21{ }^{8}$ Department of Marine Biology and Environmental Sciences, Faculty of Agriculture, University

22 of Miyazaki, Miyazaki 889-2192, Japan

Corresponding Author:

26 Nina Yasuda

27 University of Miyazaki, Faculty of Agriculture, Gakuen- kibanadai-nishi-1-1, Miyazaki 889-

28 2192, Japan

29 Email address: nina27@cc.miyazaki-u.ac.jp

\section{Abstract}

Background: Precious corals known as coralliid corals (Anthozoa: Octocorallia), play an important role in increasing the biodiversity of the deep sea. Currently, these corals are highly threatened because of overfishing that has been brought on by an increased demand and elevated prices for them. The deep sea precious corals Pleurocorallium elatius and Pleurocorallium konojoi are co-distributed in Japanese waters and have distinct morphological features: (1) the terminal branches of the colony form of $P$. elatius are very fine, while those of $P$. konojoi are blunt and rounded, (2) the autozooids of $P$. elatius are arranged in approximately four rows, while those of $P$. konojoi are clustered in groups. However, previous genetic analysis using 
40 mtDNA and nuclear DNA did not indicate monophyly. Therefore, it is important to clarify their 41 species status to allow for their conservation.

42 Methodology: We collected a total of 87 samples (60 of C. japonicum and 27 of $P$. konojoi) 43 from around the Ryukyu Islands and Shikoku Island, which are geographically separated by 44 approximately $1300 \mathrm{~km}$. We used a multiplexed inter-simple sequence repeat (ISSR) genotyping 45 by sequencing (MIG-seq) and obtained 223 SNPs with which to perform STRUCTURE analysis 46 and principle coordinate analysis (PCoA). In addition, two relatively polymorphic mtDNA 47 regions were sequenced and compared.

48 Results: Pleurocorallium elatius and P. konojoi share a same mtDNA haplotype, which has been 49 previously reported. However, MIG-seq analysis clearly distinguished the two species based on 50 PCoA and STRUCTURE analysis, including 5\% of species-specific fixed SNPs.

51 Conclusion: This study indicated that $P$. elatius and $P$. konojoi are different species and 52 therefore both species should be conserved separately. Our findings highlight the importance of the conservation of these two species, especially $P$. elatius, whose population has been dramatically depleted over the last 100 years. The study also demonstrated the effectiveness and robustness of MIG-seq for defining closely related octocoral species that were otherwise indistinguishable using traditional genetic markers (mtDNA and EF).

\section{Introduction}

The precious corals known as Coraliid (Anthozoa: Octocorallia), play an important role in increasing the biodiversity of the deep sea by providing a complex habitat structure, suitable for other species to live in (Roberts, Wheeler \& Freiwald, 2006). Their beautiful red and pink skeleton has drawn great attention and they have been fished for ornaments, jewelry, and currency since ancient times (Chen, 2012; Clark \& Rowden, 2009). Recent studies, however, indicated precious corals are vulnerable to overfishing because of their low fecundity and slow growth rate (Nonaka et al, 2015; Luan el al, 2013; Torrents et al, 2005). Consequently, there is an increasing need for the conservation of overexploited precious corals in order to avoid local extinction (CITES, 2007; CITES, 2009).

Species delimitation is important for conserving precious corals due to the fact that the species is the most fundamental unit for preservation (De Queiroz, 2007). However, species identification of the precious corals is sometimes difficult, especially for closely related species. The use of morphological characteristics in determining the species identification of octocorals, including precious corals, is the typical method, (Luan et al, 2013) however, variations, and high frequency of homoplasy sometimes hinder identification (Figueroa \& Baco, 2014). More recently, molecular and phylogenetic approaches using partial mitochondrial DNA (mtDNA) have emerged as useful tools to identify octocoral species (McFadden et al, 2011; Pante et al, 2012; Quattrini et al, 2014). In Coralliidae, the intergenic region 1 (IGR1) in mtDNA has a relatively high intra-species variation and is proposed as a useful marker for distinguishing between precious coral species ( $\mathrm{Tu}$, Dai \& Jeng, 2015). Although mtDNA is widely used for species delimitation, discordances with the morphological characteristics of species still remain. 
80 For example, morphologically distinct species in Hemicorallium cannot be delineated using

81

82

83

84

85

86

87

88

89

90

91

92

93

94

95

96

97

98

99

100

101

102

103

104

105

106

107

108

109

110

111

112

113

114

115

116

117

118
mtDNA (Ardila et al, 2012; Tu, Dai, \& Jeng, 2015). Indeed, the slow mtDNA evolution has long been recognized for anthozoans (Hellberg 2006; France \& Hoover, 2002). Octocoral species in particular have a slow mutation rate of mtDNA, due to octocoral-specific DNA repairing enzymes (Bilewitch \& Degnan, 2011; France \& Hoover, 2002). These facts indicate that a higher resolution genetic marker is required to elucidate the species status of closely related precious coral species.

The advent of high-throughput sequencing technology has rendered a large number of loci of non-model organisms accessible in a short time period. For example, the restriction site associated DNA sequence (RADseq) (Baird et al, 2008) has been applicable in non-model organisms to delimit closely related species of many reference taxa. In deep sea octocorals, only a few studies have applied RADseq for species delimitation. RADseq has helped fine-tune the species status of genus Chrysogorgia (Pante et al, 2015) and revealed robust species boundaries in the genus Paragorgia using the Bayesian model based-method (Herrera \& Shank 2016). Additional studies using the high-throughput technology to delineate closely related precious coral species would be helpful to reveal species boundaries.

Multiplexed inter simple sequence repeat (ISSR) genotyping by sequencing (MIG-seq) is an easy, cost-effective, novel method to obtain a moderate number of single nucleotide polymorphisms (SNPs) of non-model organisms using polymerase chain reaction (PCR) and high-throughput technology. The number of available SNPs from MIG-seq analysis is generally less than those using other techniques such as RAD-seq (Miller et al, 2007). However, MIG-seq has several advantages, namely that putatively neutral loci adjacent to microsatellite regions can be obtained and the method can be performed with small amounts and/or low-quality DNA, and is relatively easy to perform cheaply. The ISSR regions are first amplified by PCR using universal primer sets, indexed by a second PCR, and then sequenced to obtain up to a few thousand SNPs. Closely related species of Heliopora were successfully delineated using MIGseq (Richards et al, 2018), implying the effectiveness of this method for other closely related octocoral species.

Our target species, deep sea precious corals Pleurocorallium elatius and Pleurocorallium konojoi are co-distributed in Japanese waters at depths of 50 to 330 m (Nonaka \& Muzik, 2009). $P$. elatius and $P$. konojoi can be distinguished by at least two morphological features: (1) terminal branches of the colony form of $P$. elatius are very fine, while those of $P$. konojoi are blunt and rounded, (2) autozooids of $P$. elatius are arranged in approximately four rows, while those of $P$. konojoi are clustered in groups (Nonaka et al, 2012). Nevertheless, a previous molecular analysis suggested the two species shared a major haplotype at eight mtDNA regions and EF-1, indicating that the species status of these two is unclear (Tu, Dai \& Jeng, 2015). Because these two species, especially C. elatius, are facing the risk of local extinction due to overfishing (Nonaka \& Muzik, 2009), clarifying whether or not they are the same species with gene flow occurring between them is important for devising conservation strategies.

Peer] reviewing PDF | (2019:01:34731:2:0:NEW 10 Aug 2019) 
119

120

121

122

123

124

125

126

127

128

129

130

131

132

133

134

135

136

137

138

139

140

141

142

143

144

145

146

147

148

149

150

151

152

153

154

155

156

157

158

In this study, we applied MIG-seq analysis to clarify the species status of the two closely related precious corals, $P$. elatius and $P$. konojoi in order to obtain basic information for the conservation of precious coral species. We also sequenced widely used mtDNA regions for comparison with the MIG-seq results.

\section{MATERIALS AND METHODS \\ Sample collection and DNA extraction}

In total, 87 samples of $P$. elatius and P. konojoi (27 P. elatius and 60 P. konojoi) were collected from depths of 100 to $330 \mathrm{~m}$ using either a traditional coral net, underwater remotely operated vehicles (ROV), or submarines off Ryukyu, Kyushu, and Shikoku, Japan (Table 1). We collected some samples under the Kochi Prefecture sampling permit number Sa 401, Sa 412, and Sa 423 in Kochi. Samples were preserved in 90\% ethanol and genomic DNA was extracted using the hot alkaline solution method (Meeker et al, 2007) followed by ethanol precipitation (Richards et al, 2018).

\section{MtDNA analysis}

Two mtDNA regions were sequenced for $P$. elatius and $P$. konojoi: IGR1 region using primers (IGR1-Co-F and IGR1-Co-R, Tu, Dai \& Jeng, 2015) and putative mitochondrial DNA mismatch repairing gene (mitochondrial mutS-like protein) regions (MSH-Co-F and MSH-Co-R, Tu, Dai \& Jeng, 2015) following the original protocols. Two regions were directly sequenced from both directions using Big Dye v 3.1 and the Abi3730 sequencer. All of the sequence data were manually checked using Bioedit ver. 7.0.9.0 (Hall, 1999) and then all the sequences were aligned on GENETIX ver. 12. A maximum likelihood tree using MEGA 7.0 (Kumar, Stecher \& Tamura, 2016) was constructed using concatenated mtDNA sequences. The best model was estimated using MEGA 7.0 based on the corrected Akaike Information Criterion and the Tamura 3parameter model (Tamura, 1992) was used to construct a phylogenetic tree. Confidence values for phylogenetic trees were inferred using 1000 bootstrap replicates. All mtDNA sequences obtained in this study were deposited (DDBJ Accession number: LC464485-LC464516, LC475110-LC475134).

\section{MIG-seq analysis}

We performed a MIG-seq analysis to detect genome-wide SNPs following the protocol by Suyama \& Matsuki (2015). Briefly, MIG-seq amplifies putatively neutral, anonymous genomewide ISSR regions (Gupta et al, 1994; Zietkiewicz, Rafalski \& Labuda, 1994), including a few hundred to a few thousand SNPs, using 8 pairs of multiplex ISSR primers (MIG-seq primer set 1) for the first PCR. Then the DNA libraries from each sample with a different index were pooled and sequenced using MiSeq (sequencing control software v2.0.12, Illumina) with the MiSeq Reagent v3 150 cycle kit (Illumina). Image analysis and base calling were performed using real-time analysis software v1.17.21 (Illumina). We analyzed a total of 87 individuals with 27 P. elatius and 60 P. konojoi individuals collected from the Ryukyu Islands to Shikoku Island, geographically separated by approximately $1300 \mathrm{~km}$ (Table 1). 
159

160

161

162

163

164

165

166

167

168

169

170

171

172

173

174

175

176

177

178

179

180

181

182

183

184

185

186

187

188

189

190

191

192

193

194

195

196

197

198

To eliminate low-quality reads and primer sequences from the raw data, we used the FASTXtoolkit version 0.0.14 (fastaq_quality_filter) (Gordon \& Hannon, 2012;

http://hannonlab.cshl.edu/fastx toolkit/index.html) with a fastq-quality-filter setting of -Q $33-\mathrm{q}$ $30-\mathrm{p} 40$. We removed adapter sequences for the Illmina MiSeq run from both the 5 ' end (GTCAGATCGGAAGAGCACACGTCTGAACTCCAGTCAC) and the 3' end (CAGAGATCGGAAGAGCGTCGTGTAGGGAAAGAC) using Cutadapt version 1.13 (Martin 2011), and then we excluded short reads less than $80 \mathrm{bp}$. The quality-filtered sequence data were demultiplexed and filtered through the software Stacks v1.46 (Catchen et al, 2011; Catchen et al, 2013). We used Stacks v. 1.4 (Catchen et al, 2013) to stack the reads and extract SNPs. First, we used the U-stacks with the option settings of 'minimum depth of coverage required to create a stack $(\mathrm{m})$ ' $=3$, 'maximum distance allowed between stacks $(\mathrm{M})$ ' $=1$, 'maximum distance allowed to align secondary reads to primary stacks $(\mathrm{N})$ ' $=1$, and the deleveraging (d) and removal (r) algorithms enabled. Secondly, we used the C-stacks with the option 'number of mismatches allowed between sample loci when build the catalog (n)' $=4$, followed by the Sstacks. To confirm the consistency of the results using different sets of SNPs with different amounts of missing data, we first created three different SNP sets using population software implemented in Stacks v 1.4 by restricting the data analysis to different criteria: (i) the minimum percentage of individuals required to process a locus across all data (r) was set at $70 \%$ and restricting data analysis to a single SNP per locus, (ii) $r$ was set at $50 \%$ and a single SNP per locus was used, and (iii) $r$ was set at $50 \%$ and all SNPs per locus were used. Confirming that all the results were consistent irrespective of the data sets, we only showed the results using (i) $r$, $70 \%$, and single SNP per locus. For all of the above analyses, we set the following parameters: the minimum number of populations that a locus must be present in to process a locus $(p)^{\prime}=1$, the minimum minor allele frequency required to process a nucleotide site at a locus (min_maf) = 0.01 , the maximum observed heterozygosity required to process a nucleotide site at a locus $($ max_obs_het $)=0.9$. BayeScan v 2.0 was used to detect possible SNPs under natural selection assuming two morphological species with a default setting.

A Bayesian individual-based assignment approach as implemented in STRUCTURE 2.3.4 was used to examine the genetic boundaries between $P$. elatius and $P$. konojoi individuals.

Twenty independent runs were performed in STRUCTURE using an admixture model and allele frequency correlated model without any morphological priors. Both the length of the burn-in period and the number of Markov Chain Monte Carlo analyses (MCMC) were 200,000. We estimated $\angle K$ (Evanno, Regnaut \& Goudet, 2005), the most likely number of clusters using STRUCTURE HARVESTER (Earl \& Bridgett, 2012), CLUMPAK (Kopelman et al, 2015), and DISTRUCT (Rosenberg, 2004) to summarize and visualize the STRUCTURE results. In addition, individual-based principle coordinate analysis (PCoA) was performed using GeneAlex ver. 6.5 to visualize the genetic relationship among different individuals in 2 dimensions. Data files were converted to each software using PGDspider ver 2.0.8.3(Lischer \& Excoffier, 2011).

\section{RESULTS}

Peer) reviewing PDF | (2019:01:34731:2:0:NEW 10 Aug 2019) 


\section{MtDNA analysis}

200 A total of $961 \mathrm{bp}$ (456 bp IGR1 sequences and $505 \mathrm{bp}$ MutS after trimming unreliable

201 sequences) from 25 individuals (13 P. elatius and 12 P. konojoi) were obtained and concatenated

202 to reconstruct the phylogenetic tree (Fig. 1). The genetic diversity of the concatenated sequences

203 for $P$. elatius and $P$. konojoi was low, with only 3 haplotypes with 2 polymorphic sites, including

2041 gap, uncovered across 25 sequences in the concatenated sequence. The haplotype diversity (h)

205 was 0.453 and nucleotide diversity $(\pi)$ was 0.00047 . The haplotype network analysis revealed $P$.

206 elatius and P. konojoi shared a major haplotype (Fig. 1a). Four out of 12 P. konojoi individuals

207 shared a major haplotype with $P$. elatius and all the $P$. elatius individuals shared the major

208 haplotype with $P$. konojoi. The reconstructed phylogenetic tree indicated that the two

209 morphological species are not monophyletic (Fig. 1b). P. elatius included one polymorphic site

210 and one gap site in IGR1 and no polymorphic sites in MutS regions, while All P. konojoi shared

211 a single haplotype at both loci (Table 1).

212

213

214

215

216

217

218

219

220

221

222

223

224

225

226

227

228

229

230

231

232

233

234

235

236

237

238

\section{MIG-seq analysis}

In total, 25,444,986 raw reads with an average of 292,471 reads per sample were obtained for 87 individuals by MIG-seq analysis, of which 25,241,178 reads remained after filtering out low quality reads. We obtained 16,011,472 reads with an average of 184,039 reads per individual after two step filtering. The three SNP data sets created using different criteria resulted in a different number of loci: (i) $r=0.7$ and only a single SNP per locus resulted in 223 loci, (ii) $r=$ 0.5 and only a single SNP per locus resulted in 762 SNPs, and (iii) $r$ was set at 0.5 and all SNPs per locus resulted in 2251 SNPs. All data sets indicated consistent STRUCTURE and PCoA results, regardless of the different ratio of missing data and possible linkage disequilibrium (Suppl. 1).

BayeScan indicated that all the loci were neutral (q-values $>0.05$ ). Among the 223 SNPs examined, 12 SNPs indicated species-specific alleles (fixed substitution between the species) that could be observed.

Both STRUCTURE and PCoA indicated the same patterns in all of the SNPs sets; P. elatius and $P$. konojoi are genetically distinct. Calculation of $\angle K$ of the STRUCTURE results indicated that $K=2$ best explained the data with a mean likelihood $=-5666.970$ and mean similarity score among 10 independent runs $=0.999$. Clear genetic differences corresponded with the morphological differences without any genetically admixed individuals (Fig. 2a). PCoA indicated two morphological species are clearly separated by the x-axis, which explains $18.5 \%$ of the data (Fig. 2b).

\section{DISCUSSION}

Japan has been the main exporter of the precious corals to mainland China. The increased demand of the precious corals in mainland China in recent years resulted in elevated prices for raw corals, and promoted illegal fishing and overexploitation. Precise harvest data for precious corals is currently unavailable due to the inconsistent application of CITES listings, unreported 
239 trade as personal or household effects, and illegal and unreported trade. Under these

240 circumstances, having species-specific customs codes for CITES would be necessary to obtain

241 accurate trade data for conservation (Shiraishi, 2018).

242 Previously, P. elatius and P. konojoi were considered morphologically different; traditional

243 genetic analysis using mtDNA and nuclear elongation factor region (Tu, Dai \& Jeng, 2015) did

244 not indicate their monophyly and thus they have been genetically indistinguishable. In the

245 present study, we confirmed that $P$. elatius and $P$. konojoi share a same haplotype (Fig. 1), which

246 is consistent with the previous study. MIG-seq analysis, however, successfully discriminated $P$.

247 elatius from P. konojoi. PCoA and STRUCTURE results indicated that the two species are

248 clearly different with no intermediate genotypes, indicating no current hybridization between the

249 two species. It is possible that extremely slow substitution rates of mtDNA of $P$. elatius and $P$.

250 konojoi resulted in non-reciprocal monophyly due to the maintenance of an ancestral shared

251 variation presented at the time of divergence. On the other hand, it is possible that a strong

252 purifying selection could encourage them to split to reciprocal monophyly in nuclear DNA, as

253 the MIG-seq analysis suggested more than 5\% of the loci showed fixed substitution between $P$.

254

255

256 elatius and P. konojoi, while BayeScan indicated all MIG-seq loci are all neutral.

257

258

259

260

261

262

263

264

265

266

267

268

269

270

271

272

273

274

275

276

277

278

This study demonstrated that $P$. elatius and $P$. konojoi are indeed different species without any hybridization or on-going gene flow consistent with morphological differences, and therefore require separate conservation management plans since $P$. elatius populations cannot be replenished by $P$. konojoi populations. $P$. elatius made up $20.6 \%$ of the total catch of precious corals in Japan from 1904 to 1920 . Currently, this has dropped to $2.0 \%$ because the biomass of P. elatius has been dramatically depleted due to overfishing (Iwasaki, 2018); thus, there is an urgent need to devise and implement conservation strategies for P. elatius.

This study highlighted the effectiveness of the MIG-seq analysis using the high throughput sequencing technology for delimiting octocoral species. This study, as well as a previous study on shallow water octocoral species, demonstrated that MIG-seq analysis can uncover genetic lineages that are undetectable when using mtDNA or ITS2 (Richards et al, 2018). MIG-seq provides a time-saving ( 3 days at shortest), simple (two PCR steps), and economical (15 US dollars per sample) approach that is applicable even for small amounts of degraded or valuable samples for SNP genotyping. Data comparison among different species or genera is easy as the MIG-seq always uses the same multiplex primer sets, which would also be suitable for nuclear barcoding among different species. Further application of MIG-seq analysis to a wider range of octocoral taxa would help to clarify the unsolved status of closely related species.

\section{CONCLUSIONS}

This study indicated that $P$. elatius and $P$. konojoi are different species and that both species should be conserved separately. The results highlighted the importance of conservation of these two species, especially P. elatius, which has been dramatically depleted in the past 100 years. 
279

280

281

282

283

284

285

286

287

288

289

290

291

292

293

294

295

296

297

298

299

300

301

302

303

304

305

306

307

308

309

310

311

312

313

314

315

316

317

318

319

Again, it does suggest that separate conservation strategies will be required for the two different morphospecies. This study also demonstrated the effectiveness and robustness of MIG-seq for delimiting closely related octocoral species that were previously indistinguishable when using traditional genetic markers (mtDNA and EF). Further application of MIG-seq analysis for a wider range of octocoral or other taxa would aid in verifying the robustness of MIG-seq analysis on species delimitation.

\section{Acknowledgements}

We are grateful to Mr. Masao Nakano, Yoshihiko Niiya, Katsuhiro Fujita for collecting samples in Kochi. We thank Mr. Hideaki Yuasa, Ms. Akemi Yoshida for their help in the analysis.

Computations were partially performed on the NIG supercomputer at ROIS National Institute of Genetics.

Fig. 1 MtDNA haplotype network considering gap regions (a) and maximum likelihood phylogenetic tree excluding gap regions (b). P. elatius (PE) is shown in pink and P. konojoi (PK) is shown in black/white. (a) The size of the circle represents the number of haplotypes found in the analysis. (b) Only the nodes with bootstrap values $(>50)$ were indicated.

Fig. 2 (a) A STRUCTURE cluster assignment result based on 223 SNPs assuming two clusters $(K=2)$. Each bar represents an individual precious coral, and colors represent inferred population ancestry. Mean (LnProb) = -5666.970 and Mean (similarity score) among 10 runs $=0.999$ (b) Individual-based Principle Coordinate Analysis results (PCoA) using 223 SNPs data obtained by MIG-seq analysis. $\mathrm{X}$ axis indicates $18.5 \%$ and $\mathrm{Y}$ axis indicates $3.52 \%$ of the data. PK and PE represent $P$. konojoi and, $P$. elatius respectively.

\section{References}

Ardila NE, Giribet G, Sánchez JA. 2012. A time-calibrated molecular phylogeny of the precious corals: reconciling discrepancies in the taxonomic classification and insights into their evolutionary history.BMC Evolutionary Biology 12: 246. doi: 10.1186/1471-2148-12-246 PMID: 23249327

Baird NA, Etter PD, Atwood TS, Currey MC, Shiver AL, Lewis ZA, Selker EU, Cresko WA, Johnson EA. 2008. Rapid SNP Discovery and Genetic Mapping Using Sequenced RAD Markers. PLoS ONE 3:e3376, doi:10.1371/journal.pone.0003376.

Bilewitch J, Degnan S. 2011. A unique horizontal gene transfer event has provided the octocoral mitochondrial genome with an active mismatch repair gene that has potential for an unusual self-contained function. BMC Evolutionary Biology 11:228. doi:10.1186/1471-2148-11-228 
320

321

322

323

324

325

326

327

328

329

330

331

332

333

334

335

336

337

338

339

340

341

342

343

344

345

346

347

348

349

350

351

352

353

354

355

356

357

358

359

360

361

362

363

364

Catchen, JM, Amores A, Hohenlohe P, Cresko W, Postlethwait JH. 2011. Stacks: building and genotyping loci de novo from short-read sequences. G3: Genes, Genomes, Genetics 1, 171-182. doi: 10.1534/g3.111.000240

Catchen J, Hohenlohe PA, Bassham S, Amores A, Cresko WA. 2013. Stacks: an analysis tool set for population genomics. Molecular ecology. 22: 3124-3140. doi: $10.1111 / \mathrm{mec} .12354$

Chen HH, Qu L, Xu Z-H, Zhu J-K, Xue H-W. 2018. EL1-like casein kinases suppress $A B A$ signaling and responses by phosphorylating and destabilizing the $A B A$ receptors PYR/PYLs in arabidopsis. Molecular Plant 11:706-719 doi: 10.1016/j.molp.2018.02.012.

Chen CS. 2012. Management of the precious coral fishery in Taiwan: Progress and perspectives. Marine Policy 36:623-629. doi: 10.1016/j.marpol.2011.10.016

CITES-Qatar. 2009. Consideration of proposals for amendment of appendices I and II. CITES CoP15 Prop. 21, p 1. Available at: https://www.cites.org/sites/default/files/eng/cop/15/prop/E-15-Prop-21.pdf (accessed 15 January 2019)

CITES-Netherlands. 2007. Inclusion of all species in the genus Corallium in Appendix II of CITES. This taxon comprises 26 closely related species. Consideration of proposals for Amendment of Appendices I and II. CITES CoP14 Inf. 36 p1 Available at: https://www.cites.org/sites/default/files/common/cop/14/inf/E14i36.pdf (accessed 15 January 2019)

Clark MR, Rowden AA. 2009. Effect of deepwater trawling on the macro-invertebrate assemblages of seamounts on the Chatham Rise, New Zealand. Deep Sea Research Part I: Oceanographic Research Papers 56:1540-1554.

Costantini F, Carlesi L, Abbiati M. 2013. Quantifying spatial genetic structuring in mesophotic populations of the precious coral Corallium rubrum. PLoS One. 8:e61546. doi:10.1371/journal.pone.0061546.

De Queiroz K. 2007. Species concepts and species delimitation. Syst Biol. 56: 879-886. $10.1080 / 10635150701701083$.

Evanno G, Regnaut S, Goudet J. 2005. Detecting the number of clusters of individuals using the software STRUCTURE: a simulation study. Molecular Ecology 14:2611-2620. doi: 10.1111/j.1365-294X.2005.02553.x

Earl DA, vonHoldt BM. 2012. STRUCTURE HARVESTER: a website and program for visualizing STRUCTURE output and implementing the Evanno method. Conservation Genetics Resources 4:359-361 doi: 10.1007/s12686-011-9548-7

France SC, Hoover IL. 2002. DNA sequences of the mitochondrial COI gene have low levels of divergence among deep-sea octocorals (Cnidaria: Anthozoa). Hydrobiology,471,149-155

Gordon A, Hannon GJ. .2012 "FASTX - Toolkit" , FASTQ/A short - reads pre - processing tools (unpublished) http://hannonlab.cshl.edu/fastx_toolkit/

Gupta M, Chyi YS, Romero-Severson J, Owen JL. 1994. Amplification of DNA markers from evolutionarily diverse genomes using single primers of simple-sequence repeats. TAG 89, 998-1006

Kopelman NM, Mayzel J, Jakobsson M, Rosenberg NA, Mayrose I. 2015. CLUMPAK: a program for identifying clustering modes and packaging population structure

Peer) reviewing PDF | (2019:01:34731:2:0:NEW 10 Aug 2019) 
365

366

367

368

369

370

371

372

373

374

375

376

377

378

379

380

381

382

383

384

385

386

387

388

389

390

391

392

393

394

395

396

397

398

399

400

401

402

403 inferences across K. Molecular Ecology Resources 15:1179-1191. doi: 10.1111/1755-0998.12387

Librado P, Rozas J. 2009. DnaSP v5: a software for comprehensive analysis of DNA polymorphism data. Bioinformatics 25:1451-1452.

doi:10.1093/bioinformatics/btp187

Lischer HE, Excoffier L. 2011. PGDSpider: an automated data conversion tool for connecting population genetics and genomics programs. Bioinformatics 28, 298299

Luan NT, Rahman MA, Maki T, Iwasaki N, Hasegawa H. 2013. Growth Characteristics and Growth Rate Estimation of Japanese Precious Corals. Journal of Experimental Marine Biology and Ecology 441: 117-125. doi.org/10.1016/j.jembe.2013.01.012

Hellberg ME. 2006. No variation and low synonymous substitution rates in coral mtDNA despite high nuclear variation. BMC Evol Biol. Mar 16;6:24. doi: 10.1186/14712148-6-24.

Hall T. 1999. BioEdit: a user-friendly biological sequence alignment editor and analysis program for Windows 95/98/NT. Nucleic Acids Symposium Series 41:95-98.

Iwasaki N. 2018. Seibutsu No Kagaku Iden 72,3:214-233 in Japanese

Martin M. 2011. Cutadapt removes adapter sequences from high-throughput sequencing reads. EMBnet.journal 17:10-12. https://doi.org/10.14806/ej.17.1.200.

McFadden CS, Benayahu Y, Pante E, Thoma JN, Nevarez PA, France SC. 2011. Limitations of mitochondrial gene barcoding in Octocorallia. Molecular Ecology Resources 11:19-31. doi: 10.1111/j.1755-0998.2010.02875.x

Meeker ND, Hutchinson SA, Ho L, Trede NS. 2007. Method for isolation of PCR-ready genomic DNA from zebrafish tissues. Biotechniques 43610-614

Miller MR, Dunham JP, Amores A, Cresko WA, Johnson EA. 2007. Rapid and costeffective polymorphism identification and genotyping using restriction site associated DNA (RAD) markers. Gen. Res. 17, 240-248

Nonaka M, Muzik K. 2009. Recent harvest records of commercially valuable precious corals in the Ryukyu Archipelago. Marine Ecology Progress Series 397:269. doi:10.1155/2012/519091

Nonaka M, Muzik K, Iwasaki N. 2012. Descriptions of two new species and designation of three neotypes of Japanese Coralliidae from recently discovered specimens that were collected by Kishinouye, introducing a statistical approach to sclerite abundance and size. Zootaxa 3428:1.

Nonaka M, Nakamura M, Tsukahara M, Reimer JD. 2012. Histological examination of precious corals from the Ryukyu Archipelago. J Mar Biol Article ID 519091:1. doi: $10.1155 / 2012 / 519091$ 
404 Pante E, France SC, Couloux A, Cruaud C, McFadden CS, Samadi S, Watling L. 2012.

405 Deepsea origin and in-situ diversification of chrysogorgiid octocorals. PLoS ONE 406 7: e38357. doi:10.1371/journal.pone.0038357

407 Quattrini AM, Etnoyer PJ, Doughty C, English L, Falco R, Remon N, Rittinghouse M, $408 \quad$ Cordes EE. 2014. A phylogenetic approach to octocoral community structure in the deep GulfofMexico. DeepSeaRes.II 99,92102.doi:10.1016/j.dsr2.2013.05.027

Pante E, Abdelkrim J, Viricel A, Gey D, France S, Boisselier M-C, Samadi S. 2014. Use of RAD sequencing for delimiting species. Heredity 114:450-459. doi:10.1038/hdy.2014.105

Richards ZT, Yasuda N, Kikuchi T, Foster T, Mitsuyuki C, Stat M, Suyama Y, Wilson NG. 2018. Integrated evidence reveals a new species in the ancient blue coral genus Heliopora (Octocorallia). Scientific Reports 8, 018-32969. doi:10.1038/s41598-018-32969-z

Roberts JM, Wheeler AJ, Freiwald A. 2006. Reefs of the deep: the biology and geology of cold-water coral ecosystems. Science 312:543-547.doi: 10.1126/science.1119861

Rosenberg NA. 2004. DISTRUCT: a program for the graphical display of population structure. Molecular Ecology Notes, 4: 137-138. doi:10.1046/j.14718286.2003.00566.x

Shiraishi H. 2018. Seeing red. Precious coral trade in Asia. Traffic report. https://www.traffic.org/site/assets/files/11127/seeing-red-vfinal-1.pdf

Suyama Y, Matsuki Y. 2015. MIG-seq:an effective PCR-based method for genome-wide single-nucleotide polymorphism genotyping using the next-generation sequencing platform.Scientific Reports 5: 16963. doi:10.1038/srep16963

Tamura K, Nei M. 1993. Estimation of the number of nucleotide substitutions in the control region of mitochondrial-DNA in humans and chimpanzees. Molecular Biology and Evolution.Vol. 10, pp. 512-526. doi:10.1093/oxfordjournals.molbev.a040023

Kumar S, Stecher G, Tamura K. 2016. MEGA 7: Molecular Evolutionary Genetics Analysis Version 7.0 for BiggerDatasets. Molecular Biology and Evolution.33:1870-1874 doi:10.1093/molbev/msw054

Torrents O, Garrabou J, Marschal C, Harmelin JG. 2005. Age and size at first reproduction in the commercially exploited red coral Corallium rubrum (L.) in the Marseilles area (France, NW Mediterranean). Biological Conservation 121:391397. doi: 10.1016/j.biocon.2004.05.010

Tu TH, Dai CF, Jeng MS. 2015. Phylogeny and systematics of deep-sea precious corals (Anthozoa: Octocorallia: Coralliidae). Molecular Phylogenetics and Evolution 84, 173-184. doi: 10.1016/j.ympev.2014.09.031

Zietkiewicz E, Rafalski A, Labuda D. 1994. Genome fingerprinting by simple sequence repeat (SSR)-anchored polymerase chain reaction amplification. Genomics 20, 176183 
Figure 1

MtDNA haplotype network considering gap regions (a) and maximum likelihood phylogenetic tree excluding gap regions (b).

P. elatius (PE) is shown in pink and $P$. konojoi (PK) is shown in black/white. (a) The size of the circle represents the number of haplotypes found in the analysis. (b) Only the nodes with bootstrap values $(>50)$ were indicated.

(a)

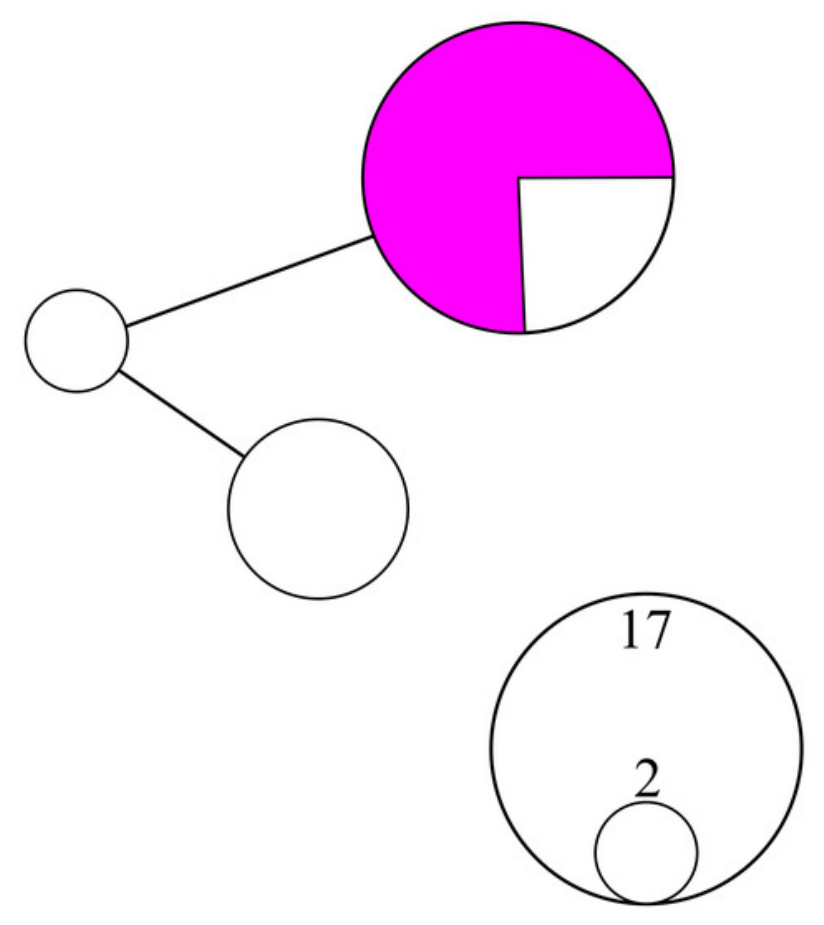

(b)

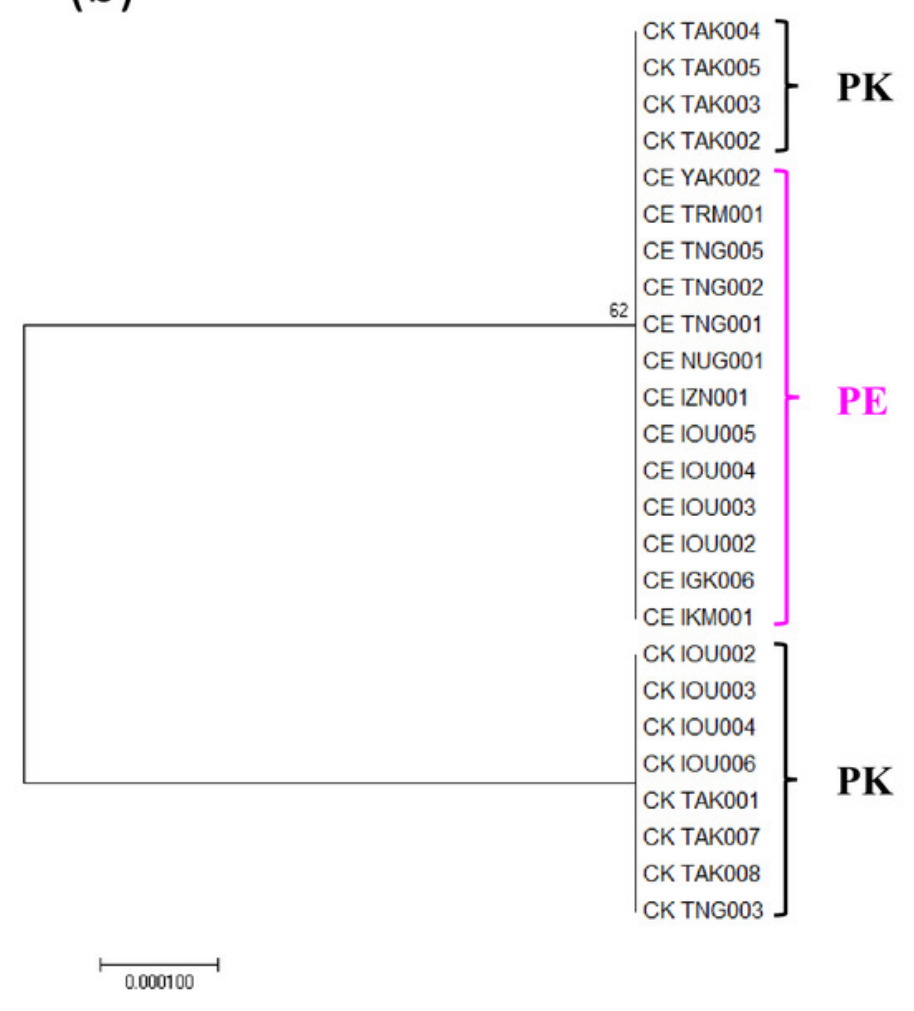


Figure 2

STRUCTURE (a) and PCoA result (b).

(a) $K=2$ with Mean (LnProb) $=-5666.970$ and Mean (similarity score) among 10 runs $=0.999$ and PCoA results using 223 SNPs data obtained by MIG-seq analysis (b). X axis indicates $18.5 \%$ and $Y$ axis indicates $3.52 \%$ of the data. PK and PE represent $P$. konojoi and, $P$. elatius respectively.

(a)

(b)

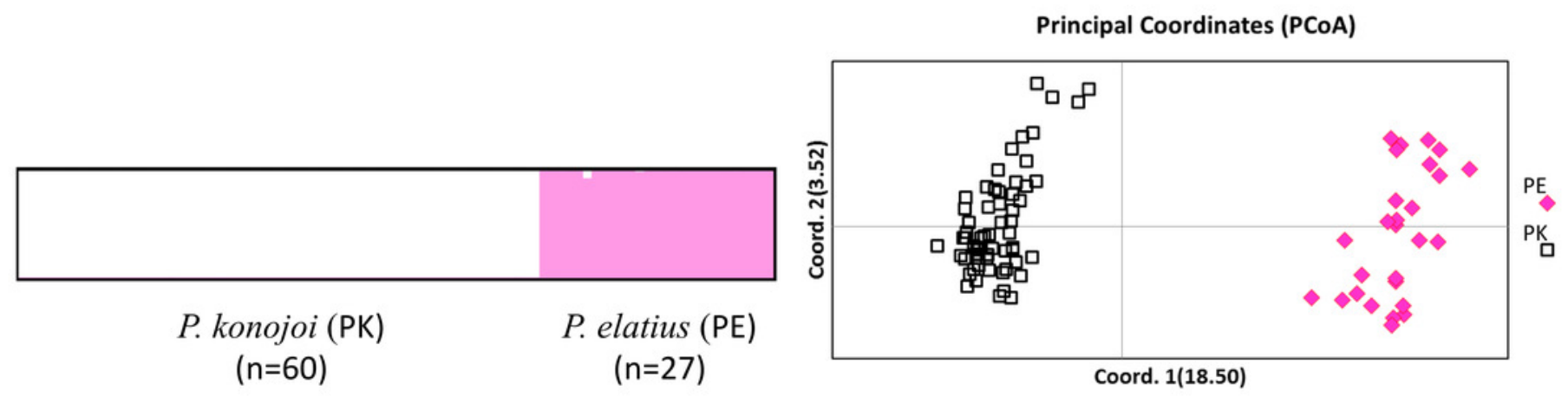




\section{Table 1 (on next page)}

Table 1 Precious coral samples used in this study 


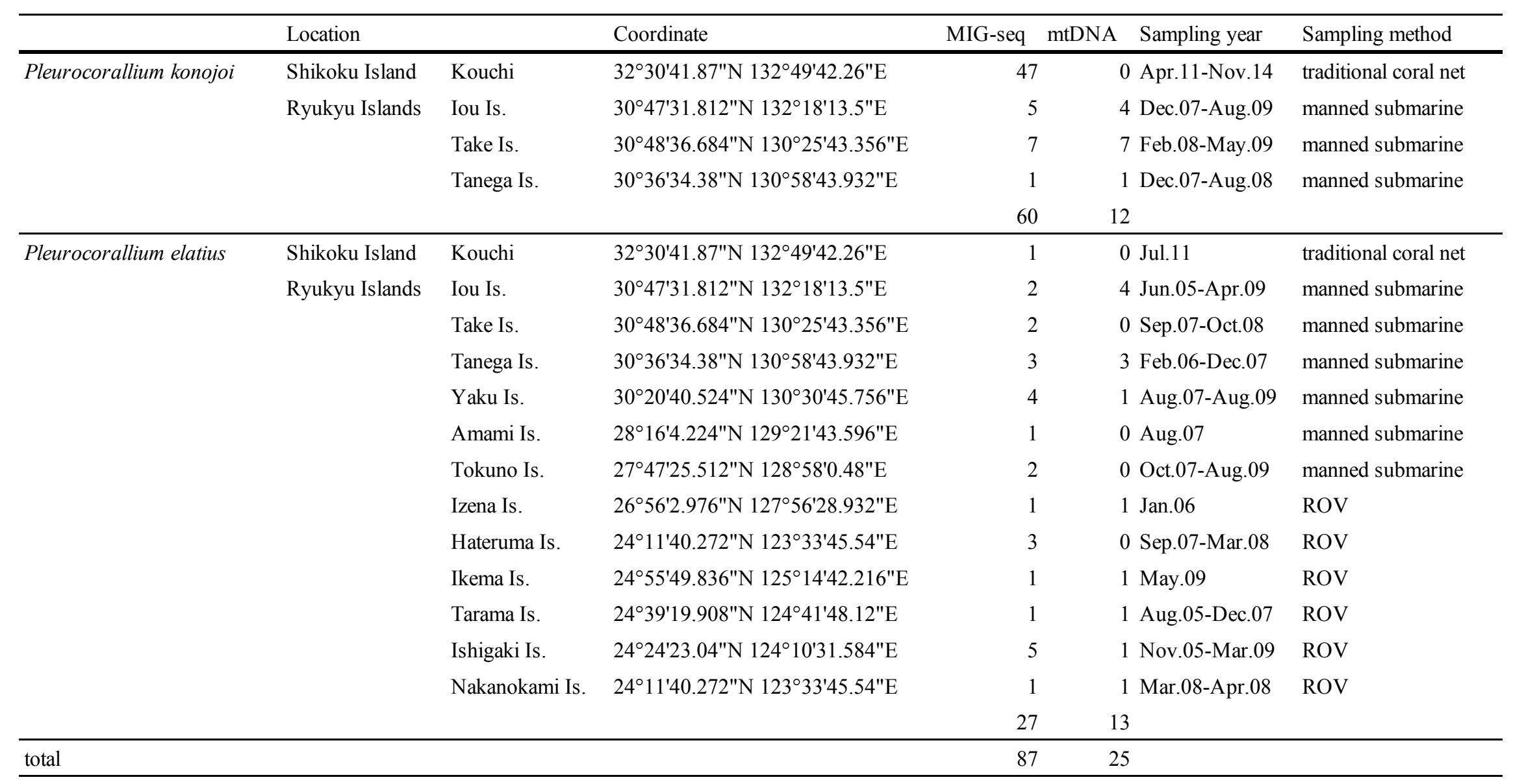

